\title{
Sex-Differences in Renal Expression of Selected Transporters and Transcription Factors in Lean and Obese Zucker Spontaneously Hypertensive Fatty Rats
}

\author{
Andrea Babelova, ${ }^{1,2}$ Birgitta C. Burckhardt, ${ }^{3}$ Waja Wegner, ${ }^{3}$ \\ Gerhard Burckhardt, ${ }^{3}$ and Maja Henjakovic ${ }^{3}$ \\ ${ }^{1}$ Institute for Cardiovascular Physiology (Physiology I), Faculty of Medicine, Goethe-University, Theodor-Stern-Kai 7, \\ 60596 Frankfurt am Main, Germany \\ ${ }^{2}$ Cancer Research Institute, Slovak Academy of Sciences, Vlarska 7, 83391 Bratislava, Slovakia \\ ${ }^{3}$ Institute for Systemic Physiology and Pathophysiology, University Medical Center Göttingen, Humboldtallee 23, \\ 37073 Göttingen, Germany
}

Correspondence should be addressed to Maja Henjakovic; maja.henjakovic@med.uni-goettingen.de

Received 1 October 2014; Revised 22 December 2014; Accepted 23 December 2014

Academic Editor: Bernard Portha

Copyright (C) 2015 Andrea Babelova et al. This is an open access article distributed under the Creative Commons Attribution License, which permits unrestricted use, distribution, and reproduction in any medium, provided the original work is properly cited.

\begin{abstract}
The aim of this study was to identify sex-dependent expression of renal transporter mRNA in lean and obese Zucker spontaneously hypertensive fatty (ZSF1) rats and to investigate the interaction of the most altered transporter, organic anion transporter 2 (Oat2), with diabetes-relevant metabolites and drugs. Higher incidence of glomerulosclerosis, tubulointerstitial fibrosis, and protein casts in Bowman's space and tubular lumen was detected by PAS staining in obese male compared to female ZSF1 rats. Real-time PCR on RNA isolated from kidney cortex revealed that Sglt1-2, Oat1-3, and Oct1 were higher expressed in kidneys of lean females. Oct2 and Mrp2 were higher expressed in obese males. Renal mRNA levels of transporters were reduced with diabetic nephropathy in females and the expression of transcription factors $\mathrm{Hnf} 1 \beta$ and $\mathrm{Hnf} 4 \alpha$ in both sexes. The highest difference between lean and obese ZSF1 rats was found for Oat2. Therefore, we have tested the interaction of human OAT2 with various substances using tritium-labeled cGMP. Human OAT2 showed no interaction with diabetes-related metabolites, diabetic drugs, and ACE-inhibitors. However, OAT2dependent uptake of cGMP was inhibited by furosemide. The strongly decreased expression of Oat 2 and other transporters in female diabetic ZSF1 rats could possibly impair renal drug excretion, for example, of furosemide.
\end{abstract}

\section{Introduction}

Diabetes mellitus is one of the most common diseases, with 346 million affected individuals worldwide in 2012 and represents the seventh leading cause of death in the United States [1]. Type 2 diabetes accounts for about $90 \%$ of all diagnosed cases [2]. More than twenty percent of patients with type 2 diabetes develop diabetic nephropathy [3]. Moreover, clinical studies reported a high prevalence of hypertension for patients in both early and late stages of this disease, which potentiates further progression of kidney damage [4]. Vice versa, the decline in kidney function contributes to elevated blood pressure in patients with type 2 diabetes [4].
Premenopausal women typically have lower blood pressure than age-matched men, possibly mediated by estradiol which appears to act as a vasodilator [5]. This is in line with a higher incidence of diabetic nephropathy associated with type 2 diabetes observed in men compared to age-matched women [6].

An unresolved issue is the association of diabetic nephropathy with expression of transport proteins responsible for renal secretion of drugs. Members of the solute carrier 22 (Slc 22) gene family, organic anion transporters (human OAT; rat and mouse Oat), and organic cation transporters (human OCT; rat and mouse Oct) are expressed in the kidneys and take up endogenous and exogenous compounds, including frequently prescribed drugs, from the blood into proximal 
TABLE 1: Blood pressure (BP), blood glucose, plasma glyceride, and urine albumin/creatinine ration in young and old male ZSF1 rats.

\begin{tabular}{|c|c|c|c|}
\hline & Lean ZSF1 & Obese ZSF1 & Reference \\
\hline $\begin{array}{l}\text { Systol. BP }(\mathrm{mmHg}) \\
8 \text { weeks of age }\end{array}$ & & $132 \pm 19$ & {$[21]$} \\
\hline $\begin{array}{l}\text { Systol. BP }(\mathrm{mmHg}) \\
20 \text { weeks of age }\end{array}$ & $156 \pm 14$ & $176 \pm 23$ & {$[21]$} \\
\hline $\begin{array}{l}\text { Diastol. BP }(\mathrm{mmHg}) \\
8 \text { weeks of age }\end{array}$ & & $88 \pm 8$ & {$[21]$} \\
\hline $\begin{array}{l}\text { Diastol. } \mathrm{BP}(\mathrm{mmHg}) \\
20 \text { weeks of age }\end{array}$ & $101 \pm 9$ & $103 \pm 11$ & {$[21]$} \\
\hline $\begin{array}{l}\text { Blood glucose }(\mathrm{mg} / \mathrm{dL}) \\
8 \text { weeks of age }\end{array}$ & $114 \pm 2.8$ & $147 \pm 3.9$ & [19] \\
\hline $\begin{array}{l}\text { Blood glucose }(\mathrm{mg} / \mathrm{dL}) \\
29 \text { weeks of age }\end{array}$ & $115 \pm 11$ & $424 \pm 37$ & {$[24]$} \\
\hline $\begin{array}{l}\text { Plasma triglyceride }(\mathrm{mg} / \mathrm{dL}) \\
8 \text { weeks of age }\end{array}$ & $49 \pm 3$ & $483 \pm 46$ & [19] \\
\hline $\begin{array}{l}\text { Plasma triglyceride }(\mathrm{mg} / \mathrm{dL}) \\
29 \text { weeks of age }\end{array}$ & $194 \pm 23$ & $5200 \pm 702$ & {$[24]$} \\
\hline $\begin{array}{l}\text { Urine albumin/creatinine ratio } \\
8 \text { weeks of age }\end{array}$ & $0.03 \pm 0.001$ & $0.23 \pm 0.04$ & [19] \\
\hline $\begin{array}{l}\text { Urine albumin/creatinine ratio } \\
16 \text { weeks of age }\end{array}$ & $0.048 \pm 0.007$ & $1.203 \pm 0.118$ & [23] \\
\hline
\end{tabular}

tubular cells [7-10]. Among antidiabetic drugs, OCT2 is involved in proximal tubular secretion of metformin, and OAT3 transports sitagliptin $[9,11]$. For rat kidneys, androgendependent expression of Oat1, Oat3, and Oct 2 and higher expression of Oat2 in females was reported, suggesting sexdependent renal drug handling at least in this species $[12,13]$.

ATP-dependent efflux transporters, multidrug resistance-associated protein 2 (Mrp2), Mrp4, and P-glycoprotein (Mdrlb) are localized in the apical membrane of renal proximal tubules and are responsible for the secretion of organic anions and cations from the proximal tubular cells into the urine [14]. Human gene promoters of OAT1, OAT2, and MRP2 are activated by the transcription factor hepatocyte nuclear factor $4 \alpha(\mathrm{HNF} 4 \alpha)$ [15-17]. Interestingly, single nucleotide polymorphisms (SNPs) associated with type 2 diabetes were found in the gene encoding HNF4 $\alpha$ [18].

The aim of this study was to identify, at the level of mRNA, potential sex- and diabetes-dependent changes of Oats, Octs, ATP-dependent efflux transporters, and the transcriptional regulators, $\operatorname{Hnf} 1 \alpha, \operatorname{Hnf} 1 \beta$, and $\operatorname{Hnf} 4 \alpha$. Additionally, the levels of sodium-dependent glucose cotransporter 1 (Sglt1) and Sglt2 were investigated. We used obese Zucker spontaneously hypertensive fatty rats (ZSF1) as an established animal model for type 2 diabetes and diabetic nephropathy. Lean ZSF1 rats served as a model-specific control.

ZSF1 rats were previously developed by crossing rat strains with different mutations in the leptin receptor gene, Zucker diabetic fatty (ZDF) rats, and spontaneously hypertensive heart failure (SHHF) rats $[19,20]$. Lean and obese ZSF1 rats had similar mean arterial blood pressure at seven weeks of age and elevated blood pressure (BP) at 20 weeks of age (Table 1) $[21,22]$. The concentration of blood glucose and plasma triglycerides were higher in obese than in lean ZSF1 rats at eight weeks of age (Table 1) [19]. However, only obese
ZSF1 rats developed type 2 diabetes with diabetic nephropathy, characterized by elevated urine albumin/creatinine ratios (Table 1) [19, 23]. Our hypothesis was that, in addition to sexdependences, the renal expression of Oats and Octs may be altered in diabetic nephropathy that might influence the renal secretion of metabolites and exogenous substances.

\section{Material and Methods}

2.1. Animals and Kidney Preservation. Kidneys from obese male and female ZSF1 (ZSF1-Lepr ${ }^{f a}$ Lepr $^{c p} / \mathrm{Crl}$ ) rats, and their lean controls, were obtained from Charles River (Stone Ridge, NY). Animals were kept in the animal facility of Charles River Laboratories under conventional housing conditions $\left(22^{\circ} \mathrm{C}, 55 \%\right.$ humidity, and $12 \mathrm{~h}$ day/night cycle) with free access to water and rat chow. Kidneys of adult (16-week-old) ZSF1 rats were removed postmortem in accordance to federal law, conserved in paraformaldehyde $(4 \%)$ or RNAlater, and shipped to our laboratory.

2.2. Morphological Study. Rat kidneys fixed in paraformaldehyde $(4 \%)$ were processed for embedding in paraffin. Serial sections $(3 \mu \mathrm{m})$ of kidneys were stained with periodic acidSchiff (PAS).

2.3. RNA Isolation. After removal of the kidney capsule, cortical slices were prepared, from which total RNA was isolated using RNeasy Mini Kit (QIAGEN, Hilden, Germany) according to the manufacturer's recommendations. Quality and quantity of the extracted RNA were determined using the Agilent 2100 Bioanalyzer (Agilent Technologies, Boeblingen, Germany) and NanoDrop ND-1000 Spectrophotometer (Thermo Scientific NanoDrop Technologies, Wilmington, 
NC), following the manufacturer's protocol. RNAs with RNA integrity number (RIN) $>8$ were used for further experiments.

2.4. cDNA Synthesis and TaqMan Real-Time PCR. Superscript II Reverse Transcriptase (Life Technologies, Darmstadt, Germany) and Oligo dT-Primers (Eurofin MWG Operon, Ebersberg, Germany) were used for reverse transcription of RNA. Genes of interest were analyzed using TaqMan Master Mix and TaqMan Gene Expression Assays (Life Technologies): Sodium-dependent glucose cotransporter 1 (Sglt1), Rn00564718_m1; Sglt2, Rn00574917_m1; Oat1, Rn00568143_ ml; Oat2, Rn00585513_ml; Oat3, Rn00580082_m1; Oct1, Rn00562250_m1; Oct2, Rn00580893_ml; Mrp2, Rn00563231_ml; Mrp4, Rn01465702_ml; P-glycoprotein (Mdrlb), Rn00561753_ml; Hnfl $\alpha$, Rn00562020_ml; Hnfl $\beta$, Rn00447453_ml; Hnf4 $\alpha$, Rn00573309_ml. The mRNA levels of hypoxanthine phosphoribosyltransferase 1 (Hprtl, Rn01527840_m1), $\beta$-actin (Rn00667869_m1), and cyclophilin A (Rn00690933_ml) were tested as housekeeping control genes for sample normalization. For all tested genes, PCR conditions were as follows: $2 \mathrm{~min}$ at $50^{\circ} \mathrm{C}$ followed by $10 \mathrm{~min}$ at $95^{\circ} \mathrm{C}$ and 40 amplification cycles $\left(95^{\circ} \mathrm{C}\right.$ for $15 \mathrm{~s}$ and $60^{\circ} \mathrm{C}$ for $60 \mathrm{~s}$ ), using Mx3000P real-time PCR cycler (Agilent Technologies). Signals detected between 35-40 amplification cycles were defined as low gene expression. The amplification efficiencies of used assays were $100 \%(+/-10 \%)$, in accordance with manufacturer's information. The real-time PCR data were analyzed as $\Delta \mathrm{Ct}=$ housekeeping gene (Hprt1)gene of interest (Figure 2), using the $2^{-\Delta \Delta \mathrm{Ct}}$ method (see Supplementary Figures in Supplementary Material available online at http://dx.doi.org/10.1155/2015/483238) [25].

2.5. Transport Studies. The uptake of cGMP in HEK293 cells stably transfected with human OAT2 (kindly provided by PortaCellTec Biosciences GmbH, Göttingen, Germany) was investigated in the absence and presence of metabolites known to be accumulated in diabetic patients and therapeutics for treatment of diabetes and hypertension. First, OAT2and vector-transfected HEK293 cells were seeded at a density of $2 \cdot 10^{5}$ cells/well in a 24 -well cell culture plate and incubated for $\sim 72 \mathrm{~h}$ in Dulbecco's modified Eagle medium-high glucose (DMEM HG, D5796, Sigma Aldrich) culture medium supplemented with $10 \%$ fetal bovine serum (number 10270, Life Technologies), 100 units/mL penicillin, and $100 \mu \mathrm{g} / \mathrm{mL}$ streptomycin (PAA Laboratories $\mathrm{GmbH}$, Austria). The cells in each well were washed with PBS and mammalian Ringer solution containing $130 \mathrm{mM} \mathrm{NaCl}, 4 \mathrm{mM} \mathrm{KCl}, 1 \mathrm{mM} \mathrm{CaCl}_{2}$, $1 \mathrm{mM} \mathrm{Mg}_{2} \mathrm{SO}_{4}, 1 \mathrm{mM} \mathrm{NaH}{ }_{2} \mathrm{PO}_{4}, 20 \mathrm{mM}$ HEPES, and $20 \mathrm{mM}$ D-glucose, $\mathrm{pH}$ 7.4. The uptake of cGMP was tested after incubation of the cells for $5 \mathrm{~min}$ with $100 \mathrm{nM}\left[{ }^{3} \mathrm{H}\right] \mathrm{cGMP}$ (PerkinElmer, Hamburg, Germany) and $9.9 \mu \mathrm{M}$ unlabeled cGMP (BioLog, Bremen, Germany), with and without potential inhibitors at $37^{\circ} \mathrm{C}$. Substances investigated for their inhibitory potential were adipic acid (Sigma Aldrich), suberic acid (Sigma Aldrich), glycolic acid (Sigma Aldrich), citric acid (Merck), 3-hydroxyisobutyrate (Fluka), cis-aconitic acid (Sigma Aldrich), homovanillic acid (Sigma Aldrich), indomethacin (Sigma Aldrich), sitagliptin (Santa Cruz Biotechnologies), miglitol (Santa Cruz Biotechnologies), captopril (Sigma Aldrich), enalapril (Sigma Aldrich), furosemide (Sigma Aldrich), and bumetanide (Sigma Aldrich). After incubation with radio-labeled cGMP and potential inhibitors, cells were washed three times with PBS at $4^{\circ} \mathrm{C}$, and cell lysis was induced by incubation for $2 \mathrm{~h}$ with $500 \mu \mathrm{L}$ of $1 \mathrm{M} \mathrm{NaOH}$. Thereafter, cell lysates were transferred to scintillations vials, $2.5 \mathrm{~mL}$ Lumasafe scintillation solution was added to each vial, and radioactivity was counted by a liquid scintillation counter (Tri-Carb 1500; PerkinElmer). Total protein concentrations were determined by the Bradford protein assay, and the cGMP uptake was calculated per milligram of total protein.

2.6. Statistical Analysis. Real-time PCR data and data of transport experiments are presented as mean \pm SEM. Statistical analysis of real-time PCR data was performed with two-way analysis of variance (ANOVA). Following two-way ANOVA, Bonferroni test was used for multiple comparison of males versus females and of lean versus obese rats (GraphPad Prism 4, version 4.03; GraphPad Software, La Jolla, CA). Data of transport experiments were statistically analyzed with twotailed unpaired $t$-test (GraphPad Prism 4). Differences were considered significant at the level of $P<0.05$.

\section{Results}

3.1. Structural Changes in the Kidneys of Lean and Obese ZSF1 Rats. Light microscopy of periodic acid-Schiff stained sections revealed differences between lean females and lean males in the structure of glomeruli and tubuli of ZSF1 rats (Figure 1, le-female, le-male). Whereas the structure of glomeruli was similar between the two sexes, the tubular basement membrane of lean males appeared to be thicker than that of lean females (arrows). Sex-differences became more obvious in obese rats where renal damage was more prominent in obese males than in obese female ZSF1 rats. In kidneys of obese male and female ZSF1 rats, glomerulosclerosis, extensive mesangial matrix accumulation, and mesangial hypercellularity were detected (Figure 1, $\$ \$ \S$ ). In obese males, in addition, a dilatation of Bowman's capsule and tuft-to-capsule adhesion was present $(\$ \$)$. Tubular injury was indicated by the thickening of tubular basement membrane, tubular dilatation with epithelial cell flattening, tubular lesions, and lumen containing protein as well as by atrophic tubuli (\#) with hyaline casts (Figure 1, \#\#).

Excessive depositions of fibronectin confirmed the development of interstitial fibrosis in kidneys of obese ZSF1 rats. Protein expression of fibronectin was higher in kidneys of lean males than of lean females (data not shown).

3.2. Differences between Lean and Obese ZSF1 Rats and SexDifferences in Renal Cortical mRNA Expression of Transporters and Transcription Factors. The housekeeping genes $\beta$-actin and cyclophilin A were differently expressed between lean and obese animals and between the sexes (data not shown). In contrast, the expression of Hprtl did not differ between 

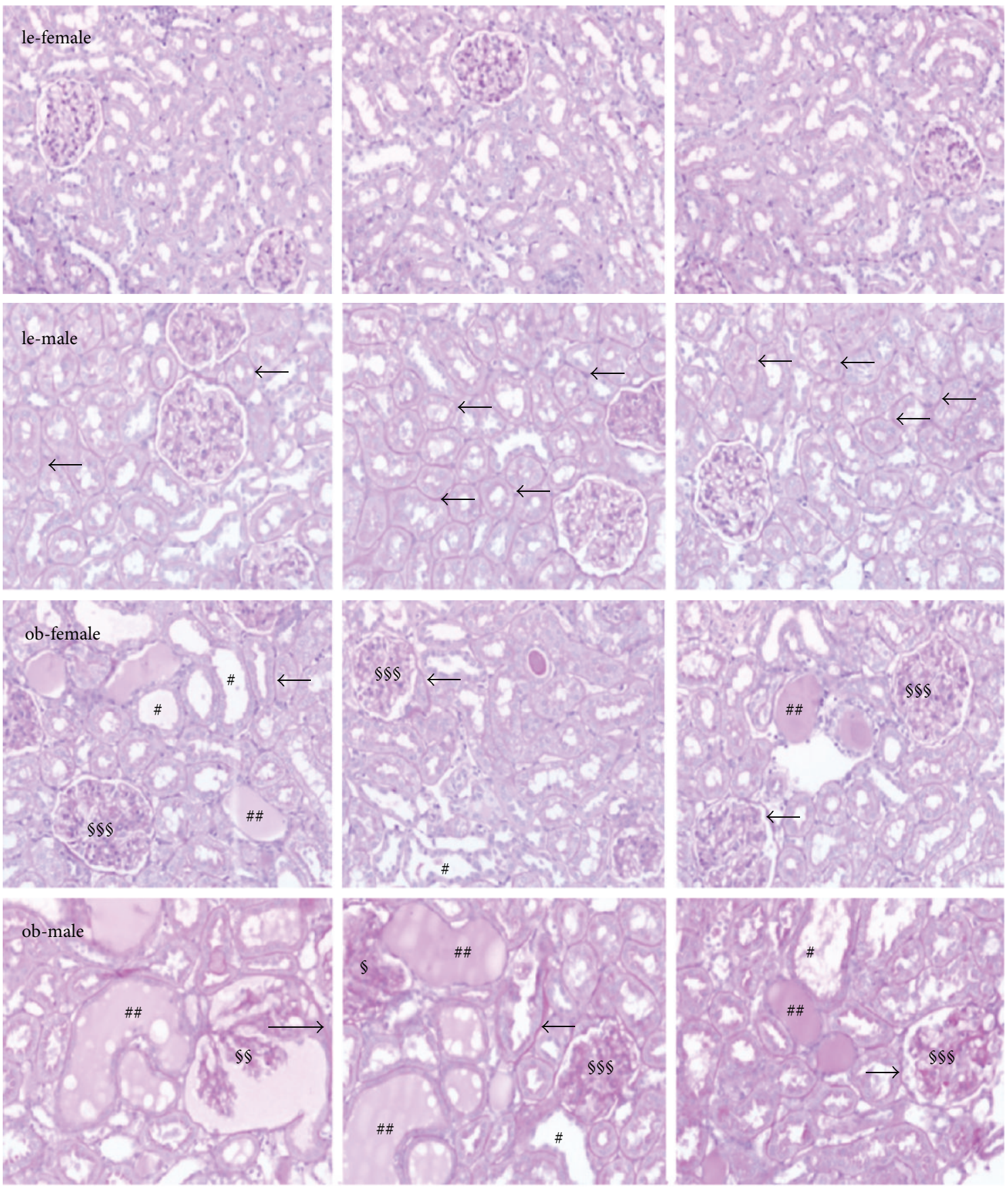

FIGURE 1: PAS staining in renal cortical slices. Renal sections of lean (le) and obese (ob) ZSF1 rats were stained with PAS and structural changes were analyzed. Examples for histological changes in renal tissue are marked as follows: \#, tubular atrophy and dilatation; \#\#, hyaline protein

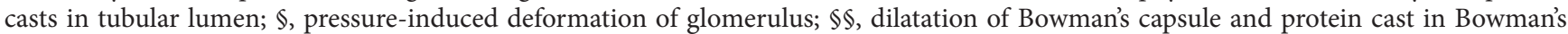
space; $\$ \$ \$$, glomerulosclerosis. The arrows mark the thickening of tubular and glomerular basement membranes. Magnification: 200x. Representative images from three different rats under each condition are shown.

experimental groups and was, therefore, used as a reference gene in our study.

Sex dependences as well as differences in mRNA expression between lean and obese rats are presented as $\Delta \mathrm{Ct}$ values in Figure 2 and are summarized as $2^{-\Delta \Delta \mathrm{Ct}}$ values in Supplementary Figures 1 and 2, respectively. In Figure 2, white bars correspond to lean and black bars to obese ZSF1 rats, respectively. Negative bars indicate that more PCR cycles were needed to reach the threshold for the gene of interest than for the reference gene Hprtl, that is, the gene of interest shows a lower expression than Hrptl. Conversely, positive bars in Figure 2 indicate a lower number of PCR cycles for the gene of interest than for Hprtl, that is, a higher gene expression as compared to Hprtl. In general, the more 

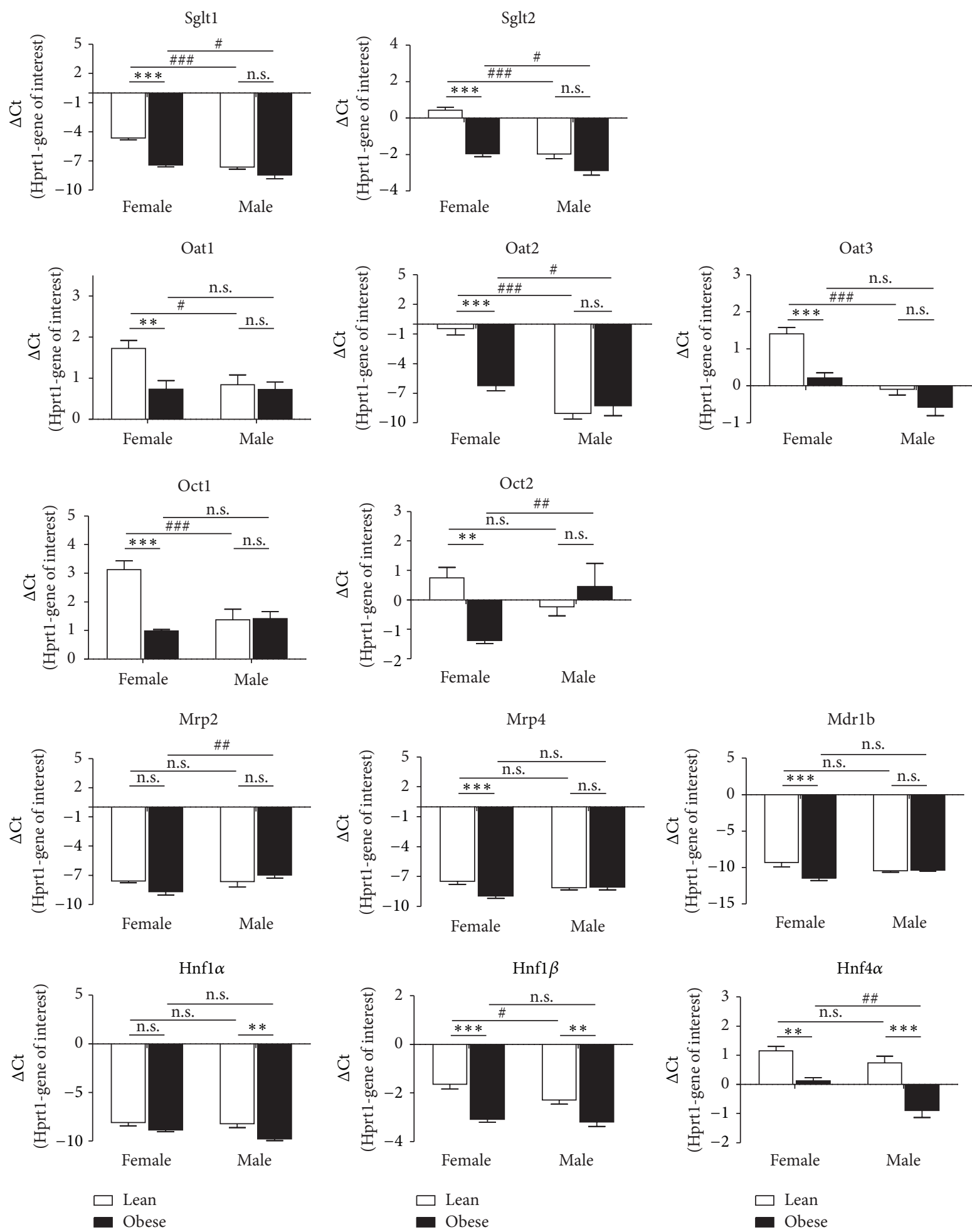

FIGURE 2: Diabetes- and sex-dependent renal gene expression in lean (le) and obese (ob) ZSF1 rats. Gene expressions were analyzed using TaqMan real-time PCR and presented as mean \pm SEM. $n=6-8$. n.s., not significant; ${ }^{* *} P<0.01$; and ${ }^{* * *} P<0.001$, for the comparison of $\Delta$ Ct values between lean and obese ZSF1 rats. ${ }^{\#} P<0.05$; ${ }^{\# \#} P<0.01$; and ${ }^{\# \# \#} P<0.001$, for comparison of $\Delta$ Ct values between females and males. 


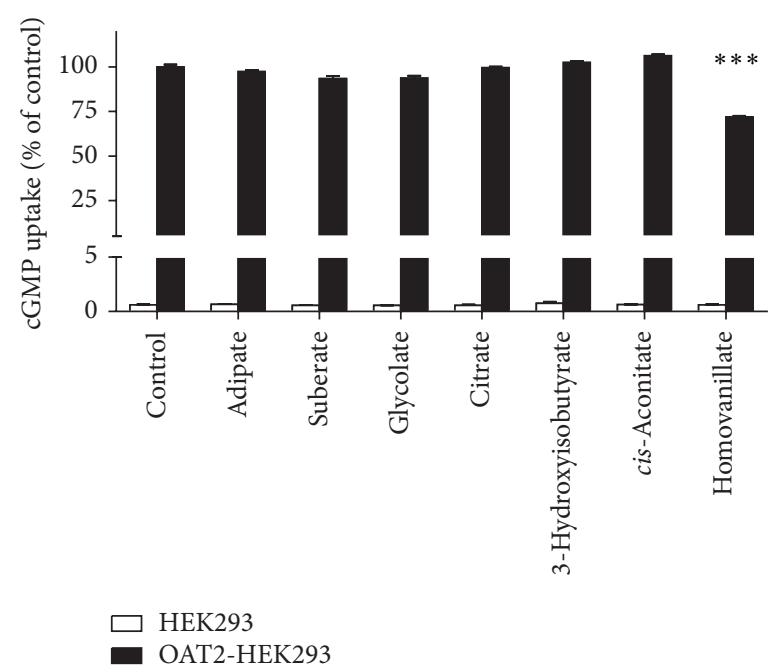

(a)

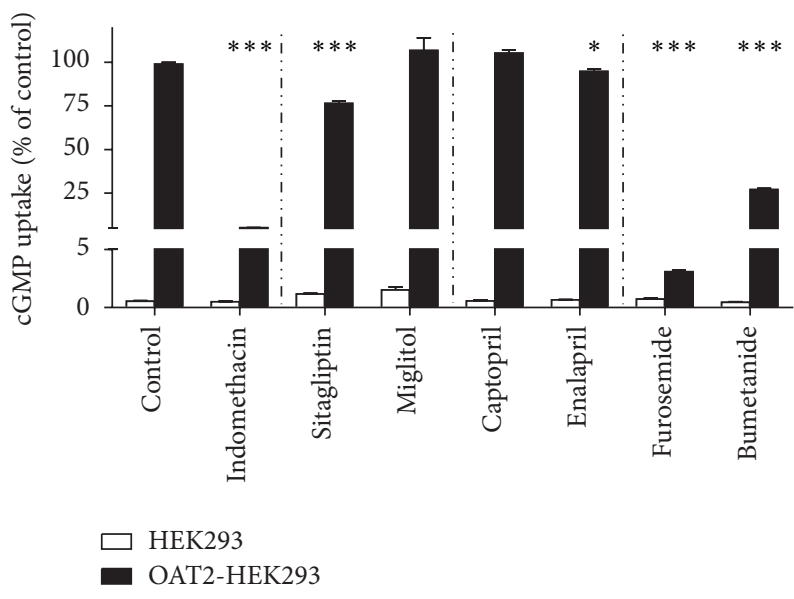

(b)

FIGURE 3: Influence of dicarboxylates, metabolites, and drugs on OAT2-mediated cGMP uptake in HEK293 cells. The uptake of cGMP was determined after 5 min incubation with $10 \mu \mathrm{M}$ cGMP $\left(0.1 \mu \mathrm{M}\left[{ }^{3} \mathrm{H}\right] \mathrm{cGMP}+9.9 \mu \mathrm{M}\right.$ unlabeled cGMP) alone (controls) or in the presence of $500 \mu \mathrm{M}$ potential inhibitors at $37^{\circ} \mathrm{C}$. (a) Dicarboxylates and metabolites; (b) drugs. Data are presented as mean \pm SEM. $n=3 .{ }^{*} P<0.05$; ${ }^{* * *} P<0.001$, compared to control.

positive (or the less negative) the $\Delta \mathrm{Ct}$ values are, the higher the expression of the gene of interest is.

In lean ZSF1 rats, mRNAs coding Sglt1 and Sglt2 were higher expressed in females, because more PCR cycles were needed to reach the threshold in males (Figure 2). In terms of $2^{-\Delta \Delta \mathrm{Ct}}$ values, sex differences amounted to $8.35 \pm 1.02$-fold for Sglt1, and to $5.51 \pm 0.68$-fold for Sglt2 (Supplementary Figure 1A). Female expression of Sglt1 and Sglt2 mRNA was retained in obese animals but was less pronounced (Figure 2). Interestingly, Sglt1 and Sglt2 expression was higher in female lean ZSF1 rats than in female obese rats. In males, no significant differences between lean and obese ZSF1 rats were observed for Sglt1 and Sglt2 (Figure 2).

Similarly, levels of Oat1, Oat2, and Oat3 were higher in kidneys of lean females than lean males (Figure 2). These sex-differences vanished for Oat1 and Oat3 in obese rats (Figure 2). In contrast, Oat2 mRNA was higher in obese female than in obese males, with a $6.70 \pm 1.58$-fold difference (Supplementary Figure 1B). In female obese ZSF1 rats, a decreased expression of Oat1, Oat2, and Oat 3 was observed in comparison with lean females (Figure 2). Interestingly, expression of Oat2 showed the strongest difference between lean and obese females. For Oat1, Oat2, and Oat 3 mRNA levels no significant differences were detected between male lean and obese ZSF1 rats (Figure 2).

In lean female ZSF1 rats, the mRNA expression level of Octl was significantly higher than in their male counterparts and this sex dependence vanished in the kidneys of obese animals (Figure 2). Renal mRNA expressions of Octl and Oct2 were significantly reduced in female obese ZSF1 rats as compared to lean females. No significant changes in Octl and Oct2 levels were detected between lean and obese male rats. In contrast, significantly higher Oct 2 expression was detected in obese males as compared to obese females (Figure 2).
Lean female rats showed a higher Mrp4 and Mdrlb expression than obese females; however, no change in Mrp2 mRNA. In male ZSF1 rats, expression of Mrp2, Mrp4, and Mdrlb remained unchanged in obese rats compared to leans (Figure 2). Renal expression of Mrp2, Mrp4, and Mdrlb showed no significant sex dependence in lean ZSF1 rats. In obese animals, Mrp2 mRNA was higher in males than in females (Figure 2).

The expression of the transcription factor Hnfl $\alpha$ was low in all tested animal groups with a detection limit beyond 35 amplification cycles. Hnfl $\beta$ and Hnf $4 \alpha$ mRNA expressions were significantly reduced in both female and male obese ZSF1 rats as compared to lean controls (Figure 2). The expression of $\operatorname{Hnfl} \beta$ was slightly higher in lean females as compared to lean males but was similar in kidneys of obese females and obese males. The transcription factor $\mathrm{Hnf} 4 \alpha$ was higher expressed in females as in males only in obese ZSF1 rats (Figure 2).

3.3. Inhibition of OAT2 Transport Function. Given the large changes in Oat2 expression, the inhibitory potential of metabolites (adipate, suberate, glycolate, citrate, 3-hydroxyisobutyrate, cis-aconitate, and homovanillate) associated with diabetic kidney disease [26, 27], drugs for treatment of diabetes (sitagliptin, miglitol), and hypertension (captopril, enalapril, furosemide, and bumetanide) on human OAT2 expressed in HEK293 cells was investigated.

The OAT2-dependent accumulation of radioactive labeled cGMP, a known substrate for OAT2 [28], was not affected by dicarboxylates adipate and suberate (Figure 3(a)). In addition, cGMP uptake was not inhibited by the metabolites glycolate, citrate, 3-hydroxyisobutyrate, and cis-aconitate. In contrast, cGMP uptake was significantly decreased in 


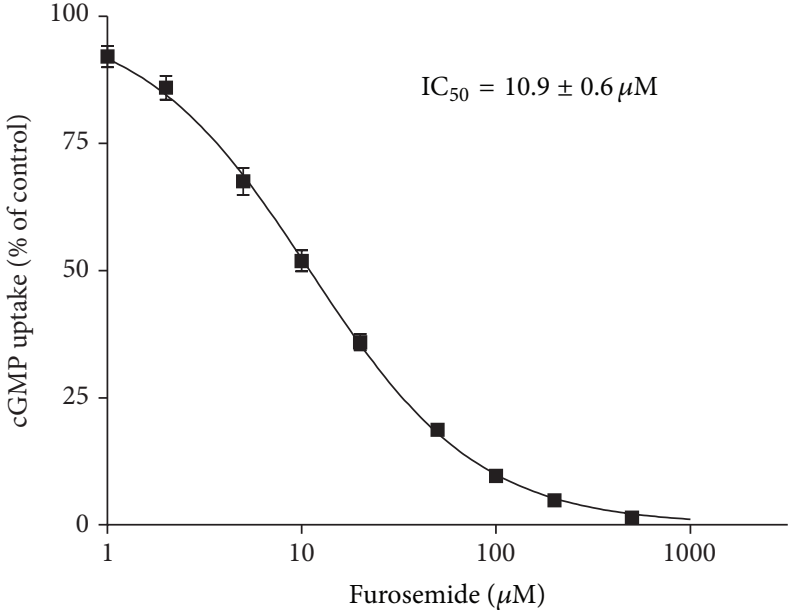

(a)

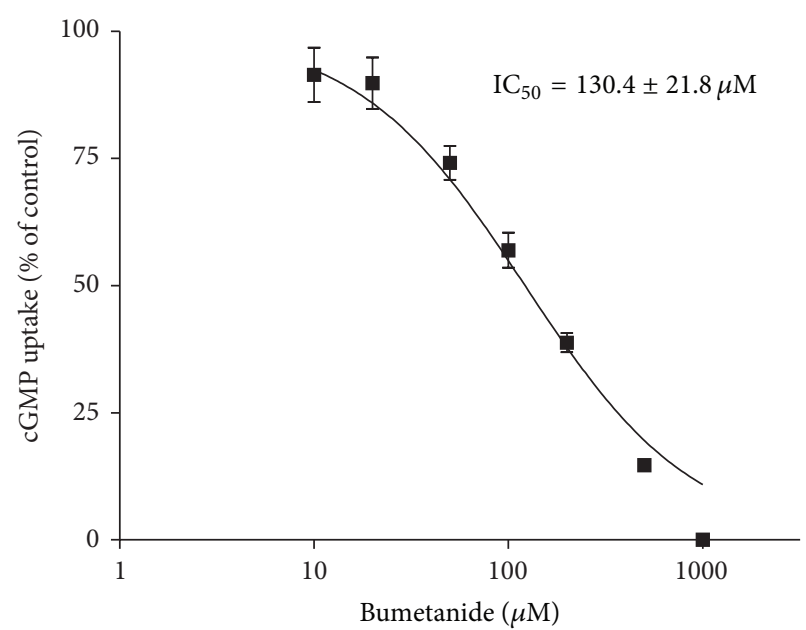

(b)

FIGURE 4: $\mathrm{IC}_{50}$ determination for the inhibition of OAT2-mediated cGMP uptake by furosemide and bumetanide. In HEK293 cells stably transfected with OAT2 or empty vector, intracellular cGMP accumulation was determined after coincubation with $10 \mu \mathrm{M}$ cGMP $(0.1 \mu \mathrm{M}$ $\left[{ }^{3} \mathrm{H}\right] \mathrm{cGMP}+9.9 \mu \mathrm{M}$ unlabeled cGMP) and 1-1000 $\mu \mathrm{M}$ furosemide (a) or 10-1000 $\mu \mathrm{M}$ bumetanide (b), respectively, for $5 \mathrm{~min}$ at $37^{\circ} \mathrm{C}$. The furosemide and bumetanide concentrations causing half-maximal inhibitory effect $\left(\mathrm{IC}_{50}\right)$ on cGMP accumulation in OAT2 expressing cells were calculated. Data are presented as mean \pm SEM. $n_{\text {furosemide }}=2-4 ; n_{\text {bumetanide }}=2$.

the presence of the dopamine metabolite homovanillate (Figure 3(a)).

OAT2 transport function was abolished by indomethacin, a verified inhibitor of OAT2-mediated cGMP uptake [29] (Figure 3(b)). The antidiabetic drug sitagliptin inhibited OAT2-mediated cGMP accumulation by approx. $25 \%$ (Figure 3(b)). Miglitol showed no effect on OAT2-dependent cGMP uptake (Figure 3(b)). No or a small significant inhibition of OAT2-dependent cGMP accumulation was observed in presence of the ACE-inhibitors captopril and enalapril (Figure 3(b)). The uptake of cGMP in OAT2expressing HEK293 cells was strongly reduced by the diuretics furosemide and bumetanide (Figure 3), showing $\mathrm{IC}_{50}$ values of $10.9 \pm 0.6 \mu \mathrm{M}$ (Figure $4(\mathrm{a})$ ) and $130.4 \pm 21.8 \mu \mathrm{M}$ (Figure 4(b)), respectively.

\section{Discussion}

Lean and obese ZSF1 rats are hypertensive, but only obese animals develop type 2 diabetes and diabetic nephropathy, exhibiting symptoms comparable to humans [19]. In this study, we show sex-dependent morphological changes in the renal cortex and in the expression of selected proximal tubular transport proteins and transcription factors in lean and obese ZSF1 rats. In addition, we investigated the impact of several metabolites found in the urine of diabetic patients and of drugs used in the treatment of diabetes and diabetes related diseases on the human organic anion transporter 2 (OAT2).

Our histological results are in line with evidence that the symptoms of diabetic renal disease, for example, renal injury, glomerulosclerosis, interstitial fibrosis, and elevated urine albumin/creatinine ratios, were more pronounced in adult male compared to female diabetic ZSF1 rats [23].
Hypertension, which was reported to be also stronger in male compared to female ZSF1 and spontaneously hypertensive heart failure (SHHF) rats was associated with higher rates of progression of glomerulosclerosis and increased fibronectin expression [30]. Thickening of tubular basement membrane observed in our study was evident not only in kidneys of obese but also in kidneys of lean male ZSF1 rats.

It has already been shown in the kidneys of Wistar rats that Sglt1 and Sglt2 proteins are higher expressed in females than in males [31]. Accordingly, in our study, lean female rats showed considerably higher mRNA levels of Sglt1 and Sglt2 than lean male rats. Recent data suggested that expression of both glucose transporters was increased in obese male Zucker rats at the age of 21 weeks [32]. We found that diabetic nephropathy in obese animals resulted in a decline in the expression of both glucose transporters. The explanation for this discrepancy is possibly the different stage of diabetic nephropathy in the Zucker obese (ZO) rat model, described in published study and in the ZSF1 rat model, used in our experiments. Our histological data confirm the published ZSF1 studies, which showed renal damage, as characteristic for diabetic nephropathy, in obese ZSF1 rats already before 21 weeks of age $[19,23]$.

In patients with diabetic nephropathy, a significantly downregulated renal OAT1 and OAT3 gene expression and impaired secretion of organic anions were observed [27]. In agreement with these human data, significant downregulation of Oat1 and Oat3 was detected in obese female ZSF1 rats compared to lean females. In contrast to published data [13], higher Oatl and Oat3 mRNA levels were detected in kidneys of female compared with male ZSF1 rats. The unexpectedly low expression of Oatl and Oat 3 in lean males could be due to structural changes as visualized by the thickening of tubular basement membrane in these animals. 
Our results showed for the first time higher expression of Octl in lean female compared with male ZSF1 rats. This finding may have some implications on drug evaluation using rats as opposed to humans, because OCT1 is not expressed in renal tubular cells of human kidneys [14]. OCT2/Oct2, wellknown for transport of the antidiabetic drug, metformin, was identified at high levels in human and rat kidneys [33]. In this study, obese males, but not lean male ZSF1 rats, showed higher Oct2 expression compared with female counterparts. In accordance with published data [34], Oct2 was decreased by diabetic nephropathy in obese females, but not in obese male ZSF1 rats.

Using another model of diabetes, Nowicki and colleagues showed increased levels of the efflux transporters, Mrp2 and Mrp4, in Western blots from whole kidneys of male rats with streptozotocin-induced type 2 diabetes [34]. We found in renal cortex no changes for Mrp2 and decreased Mrp4 mRNA levels in obese ZSF1 females compared to lean controls. Similarly, Mdrlb expression was decreased by diabetic nephropathy in our female obese rats. No changes were observed between obese and lean male ZSF1 rats. Additionally, no sex-differences were found for Mrp2, Mrp4, and Mdrlb expressions in lean animals, which is in line with already published data [35]. However, we observed higher Mrp2 expression in obese males compared with female ZSF1 rats. The reason for this sex-dependent Mrp2 expression in ZSF1 rat strain remains to be clarified.

The expression of Sglt1, Sglt2, Oct1, Oat1, and Oat3 was shown to be transcriptionally regulated by $\mathrm{Hnfl} \alpha$ homodimers and Hnfl $\alpha / \beta$ heterodimers [36-39]. Low content of Hnfl $\alpha$ mRNA was detected in renal cortex of ZSF1 rats. Thus, it appears unlikely that $\mathrm{Hnfl} \alpha$ plays a dominant role in the transcriptional regulation of transporter mRNAs. The mRNA level of $\operatorname{Hnfl} \beta$ was significantly decreased in obese ZSF1 rats compared with their lean counterparts. Furthermore, the promoters of rat Oat1, Oat3, and Octl can be activated by $\mathrm{Hnf} 4 \alpha$ [40]. The expression of $\mathrm{Hnf} 4 \alpha$ was significantly higher in obese females than in obese male ZSF1 rats and was decreased in both sexes in comparison to lean controls. HNF4 $\alpha$ has been found to be suppressed in kidneys of patients with diabetic nephropathy as well as diabetic Zucker diabetic fatty (ZDF) rats, a model for diabetic nephropathy different from that used in this study [41]. Our data showed a decline in $\mathrm{Hnf} 4 \alpha$ expression in male and female obese diabetic ZSF1 rats which is in accordance with published results [41]. The observed sex-dependent Hnf4 $\alpha$ expression in obese ZSF1 rats was possibly induced by a higher degree of diabetic nephropathy in male animals as compared to females. Interestingly, despite of reduced $\mathrm{Hnf} 4 \alpha$ expression in obese male rats, there was no change in expression of renal transporters potentially regulated by $\mathrm{Hnf} 4 \alpha$ compared to the lean animals. Given the fact that lean males exhibited a lower transporter expression compared to lean females, we assume that obesity or diabetes induced further reduction in $\mathrm{Hnf} 4 \alpha$ was not sufficient to decrease transporter expression even more.

In accordance with our results, higher renal Oat 2 expression was found in females than in males [13, 42]. Diabetic nephropathy significantly decreased Oat2 mRNA in renal cortex of obese female ZSF1 rats. Decreased Oat2 mRNA in female animals is probably the consequence of a higher degree of renal damage in kidneys of obese ZSF1 rats. In males, Oat2 mRNA expression was already very low in lean animals and did not decrease further by diabetes. This result is contradictory to previously published data, which showed increased levels of Oat 2 protein in whole kidneys of male diabetic Sprague-Dawley rats [34]. It remains to be clarified why Oat2 expression is different in diabetic kidneys of different rat strains.

In human kidneys, OAT2 is located to the basolateral membrane of proximal tubule cells [14] and is, hence, involved in the uptake of metabolites and drugs from the blood into proximal tubule cells. The renal excretion of adipate and suberate is known to be increased in ketotic episodes of diabetes [26], but neither adipate nor suberate affected OAT2, suggesting no significant role of OAT2 in excretion of these anions in diabetes. The concentrations of glycolate, citrate, 3-hydroxyisoburyrate, cis-aconitate, and homovanillate were decreased in the urine of patients with diabetic kidney disease [27]. Our experiments excluded an interaction of these metabolites with OAT2, because only homovanillate was able to inhibit cGMP uptake, but this inhibition was very weak.

The weak inhibition of OAT2-mediated cGMP uptake by high concentrations of sitagliptin and enalapril and the absence of any effect of miglitol and captopril on OAT2 function indicate that OAT2 most likely does not contribute to renal excretion of these antidiabetic and antihypertensive drugs. On the other hand, the loop diuretics furosemide and bumetanide inhibited significantly cGMP uptake by OAT2, in our study. The plasma half-life of furosemide was prolonged in patients with renal insufficiency [43]. For example, patients with mild acute kidney injury (AKI) showed a better response to furosemide than patients with severe AKI [44]. In addition, the effect of furosemide on the fractional excretion of potassium in the urine was higher in healthy volunteers compared to patients with stage III chronic kidney disease [45]. Nevertheless, loop diuretics like furosemide are appropriate in chronic kidney disease for reduction of blood pressure [46].

Our data suggest for the first time the involvement of human OAT2 in the secretion of this loop diuretic because of its high affinity $\left(\mathrm{IC}_{50} 10.9 \mu \mathrm{M}\right)$. In mouse $\mathrm{S}_{2}$ cells transfected with human OAT2, an $\mathrm{IC}_{50}$ of $603 \mu \mathrm{M}$ was published for furosemide [47]. The reason for the disagreement between our results and published data for the interaction of furosemide with human OAT2 is unclear but most likely due to the usage of different expression systems, and different radio-labeled substrates (cGMP versus $\mathrm{PGF}_{2 \alpha}$ ). Bumetanide inhibited OAT2 with moderate affinity $\left(\mathrm{IC}_{50} 130 \mu \mathrm{M}\right)$, in good agreement with previous data $\left(\mathrm{IC}_{50} 77.5 \mu \mathrm{M}\right)$ [47]. These two $\mathrm{IC}_{50}$ values are much higher than the free plasma concentration of bumetanide [47], indicating that OAT2 does not appreciably contribute to the renal excretion of bumetanide.

\section{Conclusion}

Kidneys obtained from male and female obese ZSF1 rats revealed tissue damage and significant changes in mRNA content of transporters involved in glucose absorption and drug excretion, for example, Sglt1/2, Oat1/2/3, and Oct1/2. 
These changes are most probably due to diabetes type 2 in obese ZSF1 rats. Discrete signs of damage were found in lean males, resulting from other, nondiabetic pathophysiological changes, most probably the hypertension. A number of proximal tubular transporters showed higher mRNA expression in females compared with male ZSF1 rats. Diabetes in obese animals decreased transporter expression more in female than in male rats. The highest difference was found for Oat2. Additional experiments showed for the first time the possible involvement of human OAT2 in secretion of furosemide, an often prescribed diuretic for patients suffering from diabetes, hypertension, and related kidney diseases. The altered expression of renal transporters may have an impact on sugar and drug excretion in diabetic nephropathy.

\section{Conflict of Interests}

The authors declare that there is no conflict of interests regarding the publication of this paper.

\section{Acknowledgment}

This work was supported by Deutsche Forschungsgemeinschaft (DFG; German Research Foundation; BU571/8-1; HE 6926/1-1). The funders had no role in study design, data collection and analysis, decision to publish, or preparation of the paper.

\section{References}

[1] M. Afkarian, M. C. Sachs, B. Kestenbaum et al., "Kidney disease and increased mortality risk in type 2 diabetes," Journal of the American Society of Nephrology, vol. 24, no. 2, pp. 302-308, 2013.

[2] A. Philis-Tsimikas, "Initiating basal insulin therapy in type 2 diabetes: practical steps to optimize glycemic control," The American Journal of Medicine, vol. 126, no. 9, pp. S21-S27, 2013.

[3] C. S. Fox, K. Matsushita, M. Woodward et al., "Associations of kidney disease measures with mortality and end-stage renal disease in individuals with and without diabetes: a metaanalysis," The Lancet, vol. 380, no. 9854, pp. 1662-1673, 2012.

[4] P. N. van Buren and R. Toto, "Hypertension in diabetic nephropathy: epidemiology, mechanisms, and management," Advances in Chronic Kidney Disease, vol. 18, no. 1, pp. 28-41, 2011.

[5] R. Maranon and J. F. Reckelhoff, "Sex and gender differences in control of blood pressure," Clinical Science, vol. 125, no. 7, pp. 311-318, 2013.

[6] C. Maric, "Sex, diabetes and the kidney," American Journal of Physiology-Renal Physiology, vol. 296, no. 4, pp. F680-F688, 2009.

[7] N. Anzai, Y. Kanai, and H. Endou, "Organic anion transporter family: current knowledge," Journal of Pharmacological Sciences, vol. 100, no. 5, pp. 411-426, 2006.

[8] G. Burckhardt, "Drug transport by Organic Anion Transporters (OATs)," Pharmacology and Therapeutics, vol. 136, no. 1, pp. 106130, 2012.

[9] H. Koepsell, "Polyspecific organic cation transporters and their biomedical relevance in kidney," Current Opinion in Nephrology and Hypertension, vol. 22, no. 5, pp. 533-538, 2013.
[10] L. Wang and D. H. Sweet, "Renal organic anion transporters (SLC22 family): expression, regulation, roles in toxicity, and impact on injury and disease," The AAPS Journal, vol. 15, no. 1, pp. 53-69, 2013.

[11] X. Y. Chu, K. Bleasby, J. Yabut et al., "Transport of the dipeptidyl peptidase-4 inhibitor sitagliptin by human organic anion transporter 3, organic anion transporting polypeptide $4 \mathrm{Cl}$, and multidrug resistance P-glycoprotein," Journal of Pharmacology and Experimental Therapeutics, vol. 321, no. 2, pp. 673-683, 2007.

[12] J.-I. Asaka, T. Terada, M. Okuda, T. Katsura, and K.-I. Inui, "Androgen receptor is responsible for rat organic cation transporter 2 gene regulation but not for rOCT1 and rOCT3," Pharmaceutical Research, vol. 23, no. 4, pp. 697-704, 2006.

[13] S. C. N. Buist, N. J. Cherrington, S. Choudhuri, D. P. Hartley, and C. D. Klaassen, "Gender-specific and developmental influences on the expression of rat organic anion transporters," The Journal of Pharmacology and Experimental Therapeutics, vol. 301, no. 1, pp. 145-151, 2002.

[14] K. M. Giacomini and S.-M. Huang, "Transporters in drug development and clinical pharmacology," Clinical Pharmacology and Therapeutics, vol. 94, no. 1, pp. 3-9, 2013.

[15] K. Ogasawara, T. Terada, J.-I. Asaka, T. Katsura, and K.-I. Inui, "Hepatocyte nuclear factor- $4 \alpha$ regulates the human organic anion transporter 1 gene in the kidney," The American Journal of Physiology-Renal Physiology, vol. 292, no. 6, pp. F1819-F1826, 2007.

[16] K. Popowski, J. J. Eloranta, M. Saborowski, M. Fried, P. J. Meier, and G. A. Kullak-Ublick, "The human organic anion transporter 2 gene is transactivated by hepatocyte nuclear factor- $4 \alpha$ and suppressed by bile acids," Molecular Pharmacology, vol. 67, no. 5, pp. 1629-1638, 2005.

[17] I. Qadri, L. J. Hu, M. Iwahashi, S. Al-Zuabi, L. C. Quattrochi, and F. R. Simon, "Interaction of hepatocyte nuclear factors in transcriptional regulation of tissue specific hormonal expression of human multidrug resistance-associated protein 2 (abcc2)," Toxicology and Applied Pharmacology, vol. 234, no. 3, pp. 281292, 2009.

[18] J. L. Bento, N. D. Palmer, M. Zhong et al., "Heterogeneity in gene loci associated with type 2 diabetes on human chromosome 20q13.1," Genomics, vol. 92, no. 4, pp. 226-234, 2008.

[19] V. P. Bilan, E. M. Salah, S. Bastacky et al., "Diabetic nephropathy and long-term treatment effects of rosiglitazone and enalapril in obese ZSF1 rats," Journal of Endocrinology, vol. 210, no. 3, pp. 293-308, 2011.

[20] S. P. Tofovic and E. K. Jackson, "Rat models of the metabolic syndrome," Methods in Molecular Medicine, vol. 86, pp. 29-46, 2003.

[21] S. Prabhakar, J. Starnes, S. Shi, B. Lonis, and R. Tran, "Diabetic nephropathy is associated with oxidative stress and decreased renal nitric oxide production," Journal of the American Society of Nephrology, vol. 18, no. 11, pp. 2945-2952, 2007.

[22] D. S. Tofovic, V. P. Bilan, and E. K. Jackson, "Sitagliptin augments angiotensin II-induced renal vasoconstriction in kidneys from rats with metabolic syndrome," Clinical and Experimental Pharmacology and Physiology, vol. 37, no. 7, pp. 689-691, 2010.

[23] J. H. Dominguez, P. Wu, J. W. Hawes et al., "Renal injury: similarities and differences in male and female rats with the metabolic syndrome," Kidney International, vol. 69, no. 11, pp. 1969-1976, 2006.

[24] S. P. Tofovic, H. Kusaka, C. K. Kost Jr., and S. Bastacky, "Renal function and structure in diabetic, hypertensive, obese 
ZDFxSHHF-hybrid rats," Renal Failure, vol. 22, no. 4, pp. 387406, 2000.

[25] K. J. Livak and T. D. Schmittgen, "Analysis of relative gene expression data using real-time quantitative PCR and the $2^{-\triangle \triangle C_{T}}$ method," Methods, vol. 25, no. 4, pp. 402-408, 2001.

[26] P. B. Mortensen, N. Gregersen, S. Kolvraa, and E. Christensen, "The occurrence of C6-C10 dicarboxylic acids in urine from patients and rats treated with dipropylacetate," Biochemical Medicine, vol. 24, no. 2, pp. 153-161, 1980.

[27] K. Sharma, B. Karl, A. V. Mathew et al., "Metabolomics reveals signature of mitochondrial dysfunction in diabetic kidney disease," Journal of the American Society of Nephrology, vol. 24, no. 11, pp. 1901-1912, 2013.

[28] C. D. Cropp, T. Komori, J. E. Shima et al., "Organic anion transporter 2 (SLC22A7) is a facilitative transporter of cGMP," Molecular Pharmacology, vol. 73, no. 4, pp. 1151-1158, 2008.

[29] E.-I. Lepist, X. Zhang, J. Hao et al., "Contribution of the organic anion transporter OAT2 to the renal active tubular secretion of creatinine and mechanism for serum creatinine elevations caused by cobicistat," Kidney International, vol. 86, pp. 350-357, 2014.

[30] T. Takizawa, I. Takasaki, H. Shionoiri, and M. Ishii, "Progression of glomerulosclerosis, renal hypertrophy, and an increased expression of fibronectin in the renal cortex associated with aging and salt-induced hypertension in Dahl salt-sensitive rats," Life Sciences, vol. 61, no. 16, pp. 1553-1558, 1997.

[31] L. H. Chen and P. S. Leung, "Inhibition of the sodium glucose co-transporter-2: its beneficial action and potential combination therapy for type 2 diabetes mellitus," Diabetes, Obesity and Metabolism, vol. 15, no. 5, pp. 392-402, 2013.

[32] N. M. Tabatabai, M. Sharma, S. S. Blumenthal, and D. H. Petering, "Enhanced expressions of sodium-glucose cotransporters in the kidneys of diabetic Zucker rats," Diabetes Research and Clinical Practice, vol. 83, no. 1, pp. e27-e30, 2009.

[33] N. Kimura, S. Masuda, Y. Tanihara et al., "Metformin is a superior substrate for renal organic cation transporter OCT2 rather than hepatic OCT1," Drug metabolism and Pharmacokinetics, vol. 20, no. 5, pp. 379-386, 2005.

[34] M. T. Nowicki, L. M. Aleksunes, S. P. Sawant, A. V. Dnyanmote, H. M. Mehendale, and J. E. Manautou, "Renal and hepatic transporter expression in type 2 diabetic rats," Drug Metabolism Letters, vol. 2, no. 1, pp. 11-17, 2008.

[35] H. Lu and C. Klaassen, "Gender differences in mRNA expression of ATP-binding cassette efflux and bile acid transporters in kidney, liver, and intestine of 5/6 nephrectomized rats," Drug Metabolism and Disposition, vol. 36, no. 1, pp. 16-23, 2008.

[36] L. Jin, R. Kikuchi, T. Saji, H. Kusuhara, and Y. Sugiyama, "Regulation of tissue-specific expression of renal organic anion transporters by hepatocyte nuclear factor $1 \alpha / \beta$ and DNA methylation," Journal of Pharmacology and Experimental Therapeutics, vol. 340, no. 3, pp. 648-655, 2012.

[37] R. Kekuda, P. Saha, and U. Sundaram, "Role of Sp1 and HNF1 transcription factors in SGLT1 regulation during chronic intestinal inflammation," American Journal of PhysiologyGastrointestinal and Liver Physiology, vol. 294, no. 6, pp. G1354G1361, 2008.

[38] V. P. O’Brien, K. Bokelmann, J. Ramírez et al., "Hepatocyte nuclear factor 1 regulates the expression of the organic cation transporter 1 via binding to an evolutionary conserved region in intron 1 of the OCT1 Gene," The Journal of Pharmacology and Experimental Therapeutics, vol. 347, no. 1, pp. 181-192, 2013.
[39] M. Pontoglio, D. Prié, C. Cheret et al., "HNF1alpha controls renal glucose reabsorption in mouse and man," EMBO Reports, vol. 1, no. 4, pp. 359-365, 2000.

[40] T. F. Gallegos, G. Martovetsky, V. Kouznetsova, K. T. Bush, and S. K. Nigam, "Organic anion and cation slc22 "drug" transporter (oat1, oat3, and oct1) regulation during development and maturation of the kidney proximal tubule," PLoS ONE, vol. 7, no. 7, Article ID e40796, 2012.

[41] M. Niehof and J. Borlak, "HNF4 $\alpha$ and the Ca-channel TRPC1 are novel disease candidate genes in diabetic nephropathy," Diabetes, vol. 57, no. 4, pp. 1069-1077, 2008.

[42] M. Ljubojevic, D. Balen, D. Breljak et al., "Renal expression of organic anion transporter OAT2 in rats and mice is regulated by sex hormones," American Journal of Physiology-Renal Physiology, vol. 292, no. 1, pp. F361-F372, 2007.

[43] D. C. Brater, "Diuretic therapy," The New England Journal of Medicine, vol. 339, no. 6, pp. 387-395, 1998.

[44] K. M. Ho and B. M. Power, "Benefits and risks of furosemide in acute kidney injury," Anaesthesia, vol. 65, no. 3, pp. 283-293, 2010.

[45] C. G. Musso, M. Navarro, C. Mombelli et al., "Furosemide test in stage III-chronic kidney disease and kidney transplant patients on tacrolimus," International Urology and Nephrology, vol. 45, no. 5, pp. 1471-1474, 2013.

[46] N. Vasavada, C. Saha, and R. Agarwal, "A double-blind randomized crossover trial of two loop diuretics in chronic kidney disease," Kidney International, vol. 64, no. 2, pp. 632-640, 2003.

[47] H. Hasannejad, M. Takeda, K. Taki et al., "Interactions of human organic anion transporters with diuretics," Journal of Pharmacology and Experimental Therapeutics, vol. 308, no. 3, pp. 1021-1029, 2004. 


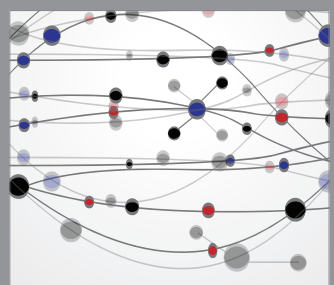

The Scientific World Journal
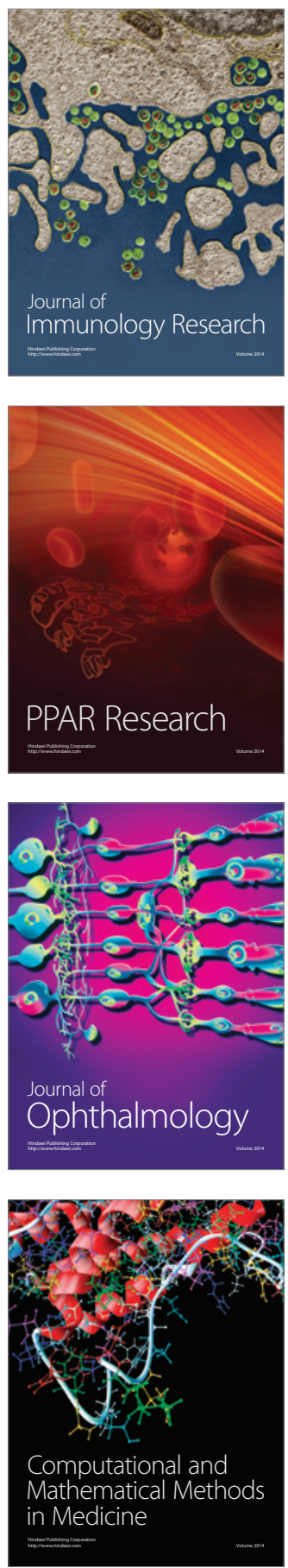

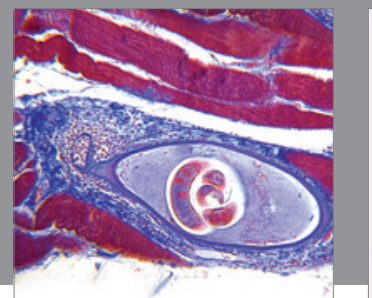

Gastroenterology

Research and Practice
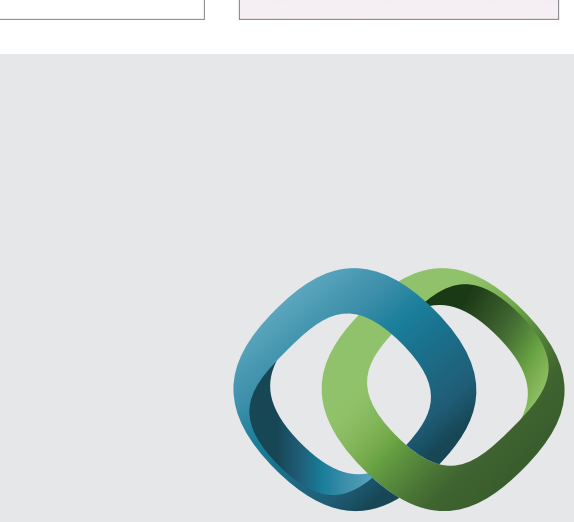

\section{Hindawi}

Submit your manuscripts at

http://www.hindawi.com
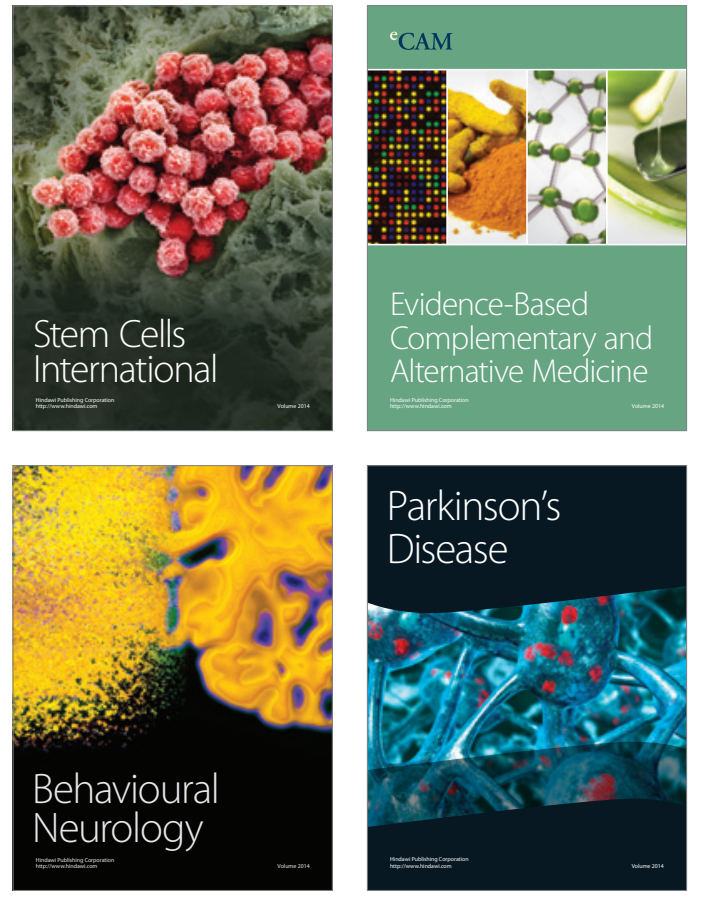
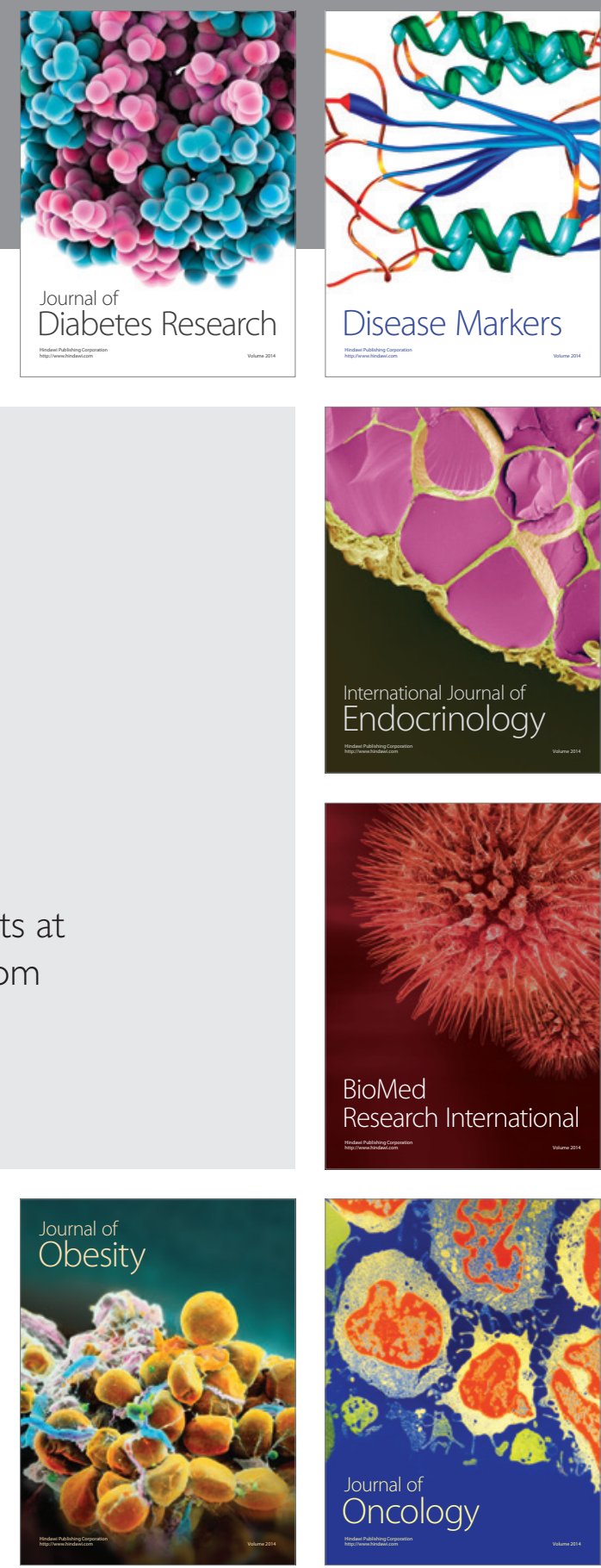

Disease Markers
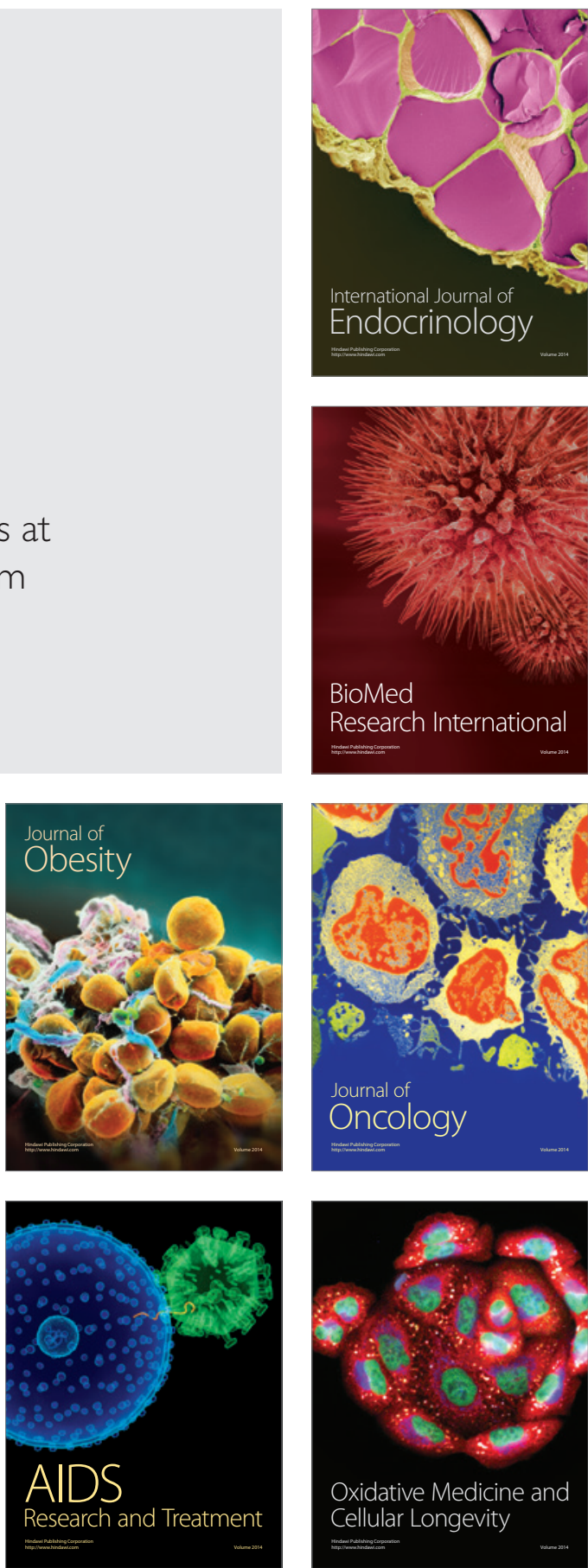